\title{
Coherent X-ray imaging at ID16A: status and plans
}

\author{
D. Karpov ${ }^{1}$, F. Monaco ${ }^{1}$, S. Bohic ${ }^{1,2}$, M. Salome ${ }^{1}$, V. Favre-Nicolin ${ }^{1}$, A. Pacureanu ${ }^{1}$, and P. Cloetens ${ }^{1}$ \\ ${ }^{1}$ European Synchrotron Radiation Facility, 71 Avenue des Martyrs, Grenoble, France, \\ ${ }^{2}$ Inserm UA7 STROBE, Universite Grenoble Alpes, 71 Avenue des Martyrs, Grenoble, France
}

dmitry.karpov@esrf.fr

High-resolution imaging of 3D structure and elemental composition is critical for studies ranging from biology to materials science. ID16A is well up to the challenge with its established record in hard X-ray phase and fluorescence imaging. Two phase imaging modalities are routinely offered at the beamline: holography that explores longitudinal diversity and near-field ptychography that explores the transverse diversity. The recent upgrade of ESRF further improves the beamline performance through increased flux and coherence and reduced spectral bandwidth.

For phase imaging the improvements of the source can lead to higher resolution and better data quality (particularly in the near-field ptychography regime). It will also directly benefit X-ray fluorescence imaging where higher flux translates directly into increased elemental sensitivity and reduced acquisition times, allowing to survey more samples resulting in the higher statistical significance of the results.

In this poster, we will discuss the status of the beamline after the transition to the ESRF-EBS and its exciting implications for the user community. We will also discuss immediate plans in the beamline development where new collaborations with the users will be mutually beneficial.

Keywords: x-ray holographic nano-tomography; ptychographic x-ray computed tomography; x-ray fluorescence tomography 